\title{
Sesgo de búsqueda y producción científica nacional en recursos humanos en salud
}

\section{Bias in literature search and national scientific production in health human resources}

\author{
Pedro Jesús Mendoza-Arana ${ }^{1}$ \\ ${ }^{1}$ Profesor Principal, Medicina Preventiva y Salud Pública, Facultad de Medicina, UNMSM, Lima, Perú; Académico Asociado, Academia Nacional de Medicina, Lima, Perú
}

DOI: http://dx.doi.org/10.15381/ anales.v78i2.13225

\section{Correspondencia: \\ Pedro Jesús Mendoza-Arana pedro.mendoza.arana@gmail.com}

Recibida: 30 abril 2017

Aceptada: 12 mayo 2017

Conflictos de interés: Ninguno.

Citar como: Mendoza Arana P. Sesgo de búsqueda y producción científica nacional en recursos humanos en Salud. Carta al Editor. An Fac med. 2017;78(2):233. DOI: http://dx.doi.org/10.15381/ anales.v78i2.13225

\section{Sr. Editor}

En el artículo de Franco Romaní y colaboradores, sobre la producción científica referida a las agendas nacionales de investigación en el Perú 2011-2014 ${ }^{(1)}$, se concluye que el área de investigación en Recursos Humanos en Salud es el área menos estudiada, con solo 4 artículos originales publicados, es decir, 1,8\% de los artículos identificados en el periodo de estudio.

Se señala asimismo como una limitación del estudio que este no refleja toda la producción peruana, sino solo los artículos incluidos en las bases de datos empleadas: SCOPUS, LILACS, y LIPECS. Entre las 31 revistas con dos o más artículos originales, solo hay tres revistas peruanas: La Revista Peruana de Medicina Experimental y Salud Pública, Horizonte Médico, y Acta Médica Peruana.

En el periodo 2011-2014, la revista Anales de la Facultad de Medicina, publicó tres artículos originales claramente referidos a los Recursos Humanos en Salud (2-4). Anales también está indizada en LILACS, por lo que estos tres artículos debieron aparecer. Una posible explicación para ello es el empleo de las palabras de búsqueda empleados por los autores: 'Health Manpower' y 'Recursos Humanos en Salud'. En los tres artículos en mención, se empleó solo 'recursos humanos' en uno de ellos (3) y otras palabras clave en los dos restantes. Sin embargo, la lectura de sus resúmenes demuestra que se trata de artículos originales y que abordan temas claros de la prioridad de investigación mencionada. Es más, en uno de ellos ${ }^{(2)}$ se aprecia que un conjunto de investigadores han desarrollado líneas de investigación sostenidas al respecto, pero usualmente entendidas más como investigación en educación médica, o investigación educacional y, por ende, involuntariamente invisibilizada por los propios autores.

El sesgo de publicación, o publication bias, ha sido largamente reconocido y estudiado. Sin embargo, en este caso lo que identificamos es un 'sesgo de búsqueda', es decir, el resultado de emplear motores de búsqueda que usan palabras específicas y ofrecen rápidos resultados en la búsqueda en extensas bases de datos. También nos lleva a la reflexión de que los autores debemos ser juiciosos al definir las palabras clave que damos a nuestros artículos, pues en lugar de ayudar a su fácil identificación, podemos estar contribuyendo a su invisibilidad como aparentemente sucede en este caso.

\section{REFERENCIAS BIBLIOGRÁFICAS}

1. Romani Romani FR, Roque Henríquez J, Vásquez Loarte T, Mormontoy Calvo H, Vásquez Sopiopuco H. Análisis bibliométrico de la producción científica peruana sobre las agendas nacionales de investigación en el Perú 2011-2014. An Fac med. 2016;77(3):241-9.

2. Mendoza P. Investigación en educación médica en la Facultad de Medicina de San Fernando: una perspectiva sistémica. An Fac med. 2012;73(1):55-61.

3. Pereyra-Elías R, Mayta-Tristán P, Cassana A, Mazones-Holguin E. Diferencias según género en la intención de emigración en médicos recién egresados. An Fac med. 2013;74(3):211-6.

4. Mendoza Arana PJ, Arzaya Mendoza MJ, Delgado Cruz DA. Vias para la articulación de la investigación en educación médica en la Facultad de Medicina de San Fernando con la investigación en recursos humanos en el Perú. An Fac med. 2014;75(1):49-55.

\section{NOTA DEL EDITOR}

La carta al Editor fue remitida a los autores del artículo aludido, obteniéndose la siguiente respuesta. 